\title{
A COMPARISON OF SURFACE SLOPES EXTRACTED FROM ICESAT WAVEFORM DATA AND HIGH RESOLUTION DEM
}

\author{
Huan Xie ${ }^{1, *}$, Hong Tang ${ }^{1}$, Wenjia Du ${ }^{1}$, Xiaohua Tong ${ }^{1}$ \\ ${ }^{1}$ College of Surveying and Geo-informatics, Tongji University, 1239 Siping Road, Shanghai 200092, \\ P. R. China \\ *Email: huanxie@tongji.edu.cn
}

\begin{abstract}
Surface slope is an important topographic variable, accurate surface slope can support many research appliacations. Large footprint full waveform data has been used to estimate the surface slope and performes well. In this paper, surface slope within laser footprint is calculated using the Ice, Cloud, and land Elevation Satellite (ICESat) Geoscience Laser Altimeter System (GLAS) full waveform data and a high resolution Digital Elevation Model (REMA, the Reference Elevation Model of Antarctica). A comparison is done between two extracted surface slopes, the results show that the slopes extracted from full waveform data are close to slopes extracted from DEM, and the width of waveform can be used to extract surface slope in moderately sloping surface.
\end{abstract}

\section{INTRODUCTION}

The Ice, Cloud, and land Elevation Satellite (ICESat) was designed to measure ice sheet mass balance, change of polar climate, cloud and aerosol heights, land topography and vegetation characteristics $^{[1]}$. From 2003 to 2009, the Geoscience Laser Altimeter System (GLAS) aboard ICESat provided 15 different kinds of products around the globe, including global elevation data, global atmosphere data, global land surface altimetry data and so on. These data have been widely used in polar ice sheet change detection, biomass information extraction, land elevation changes, oceanographic and sea-ice thickness studies, cloud height determination and vertical distribution of aerosol ${ }^{[2]}$. Accurate surface slope information is important to monitor the elevation and mass changes of ice sheet using laser altimeters $^{[3]}$. For vegetation research, GLAS waveform data can be used to extract vegetation parameters such as vegetation height, fractional coverage, biomass, etc. However, the accuracy of these parameters are affected by sloping terrain, and surface slope corrections should be considered in vegetation parameters extraction ${ }^{[4]}$. The width of waveform from sloping surfaces are broader than those from flat surfaces ${ }^{[5]}$, and the echo widths can be used to estimate surface slope. In this paper, a model based on triangle geometry is used to extract the surface slope from ICESat waveform data, and the surface slope is also extracted via a high resolution DEM (REMA). Finally, a comparison between the slope extracted from the waveform data and those estimated using the REMA DEM data is presented.

\section{EXPERIMENTAL DATA}

\subsection{ICESat/GLAS data}

The National Snow and Ice Data Center (NSIDC) provides 15 Level-1 and Level-2 data products from GLAS acquired between 2003 to 2009. In this paper, we used GLAH01(version 33), GLAH05 and GLAH14 data from version 34. GLAH01 contains the echo waveform signal and other waveform shape parameters, GLAH05 provides the size, shape and orientation parameters of footprints on the Earth's surface, and GLAH14 stores position information of laser footprints.

\subsection{REMA}

REMA (The Reference Elevation Model of Antarctica) is a high resolution, time-stamped Digital Elevation Model (DEM) of Antarctica with an 8-meter spatial resolution. REMA is extracted from satellite images including WorldView-1, WorldView-2, WorldView-3, and GeoEye-1, which were acquired between 2009 and 2017. Each individual DEM was vertically registered to satellite altimetry data from Cryosat-2 and ICESat, and the absolute uncertainties are less than $1 \mathrm{~m}$ over most of its area, and the relative uncertainty is on the order of decimeters ${ }^{[6]}$.

\section{METHODOLOGY}

\subsection{Surface slope extracted from waveform}


GLAS mounted on ICESat transmits bell-shaped laser pulses and records the full return signals which we refer to as full waveform data. The transmitted bell pulse is modeled as a Gaussian function with a certain peak position, width and amplitude. After the pulse interacts with the earth surface, the peak position, width and amplitude of the echo waveform will be altered and the waveform of echo can be modeled as a single (or sum of several) Gaussian function(s) ${ }^{[7]}$. Generally, the echo waveform has a lower amplitude and a wider width when compared to the transmitted pulse due to the effects of attenuation, surface sloping and roughness ${ }^{[8]}$. This phenomenon allows a possibility for extracting surface slope from the broadened waveform.

Based on triangle geometry, the surface slope was described as follows ${ }^{[9]}$ :

$$
S=\theta+\frac{\beta_{t}}{2}+\arctan \left(t_{\Delta} \times 10^{4} / \sigma_{\Delta}\right)
$$

The surface slope $S$ is calculated by the off-nadir laser pointing direction $\theta$, the laser divergence angle $\beta_{t}$, the echo waveform width $t_{\Delta}$, and mean pulse delay $\sigma_{\Delta}$. From Ref. [9] we know that the median of $\theta$ is 0.009 degree, the $\beta_{t}$ is 0.0086 degree, the $\sigma_{\Delta}$ is $4.02 \times 10^{7}$ ns. After decompression, smoothing and Gaussian decomposition, the width of waveform $t_{\Delta}$ is extracted from preprocessed waveform. Then the surface slope is calculated by the formula above.

\subsection{Surface slope extracted from REMA}

To validate the performance of surface slope extracted from GLAS waveform data, the surface slope is also calculated using REMA (The Reference Elevation Model of Antarctica) mosaic tiles with 8-meter spatial resolution. The location of footprint is obtained from GLAH14, and the size, shape and orientation of footprint are extracted from GLAH05. The mean slope within the footprint is derived from REMA data. Figure 1 shows the footprint on DEM, $\theta$ is the azimuth of the major axis, a is the semi-major axis of footprint, and $b$ is semi-minor axis of footprint, which is calculated via semi-major axis and the footprint eccentricity.

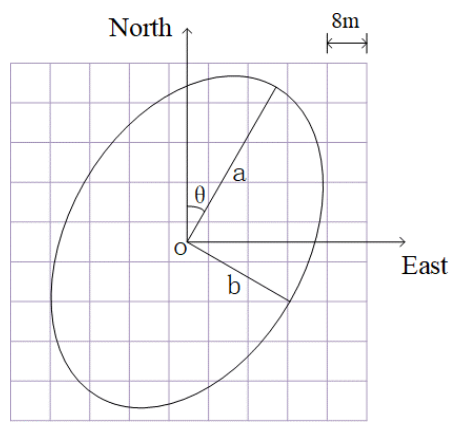

Figure 1. Footprint on DEM. The black ellipse is footprint, and the grid is DEM with 8-meter spatial resolution

\section{RESULTS AND DISCUSSIONS}

A comparison between slopes extracted from ICESat waveform data and REMA is shown in Figure 2. Quantitative statistics derived from this comparison are shown in Table 1. We find that the bias between two kinds of slopes is larger for areas where the surface slope is smaller than 1 degree. Hence, the slope extracted from waveform cannot be used in flat area. In flat area, the error may bigger than the true value.

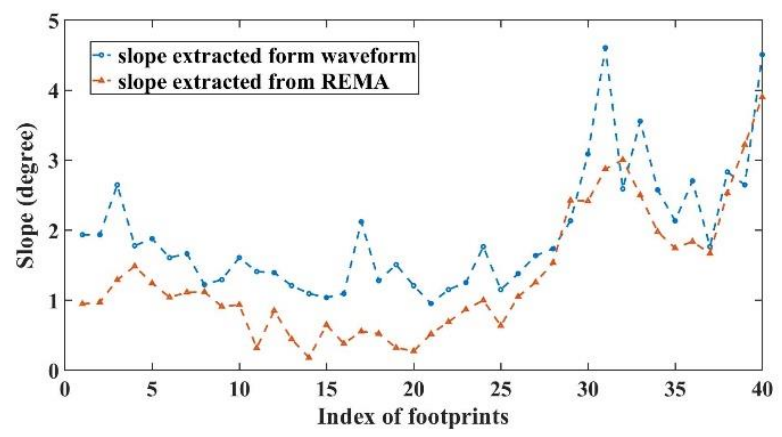

Figure 2. Comparison of waveform slopes and DEM slopes

Table 1. Statistical result of two slope types

\begin{tabular}{|c|c|c|}
\hline Slope Type & $\begin{array}{c}\text { Mean of } \\
\text { Bias }\end{array}$ & $\begin{array}{c}\text { Standard Deviation } \\
\text { of Bias }\end{array}$ \\
\hline Slope $<1^{\circ}$ & 0.76 & 0.32 \\
\hline Slope $>1^{\circ}$ & 0.55 & 0.42 \\
\hline
\end{tabular}

In order to estimate a statistical relationship between the two slope data sets, a linear regression analysis is performed. We find that the regression result is good, the RMSE is 0.4497 , and the Rsquare is 0.7429 . There are two reasons for the bias. Firstly, the width of waveform for calculating slope is not accurate. Secondly, the waveform data and 
satellite images for extracting DEM were observed in different time.

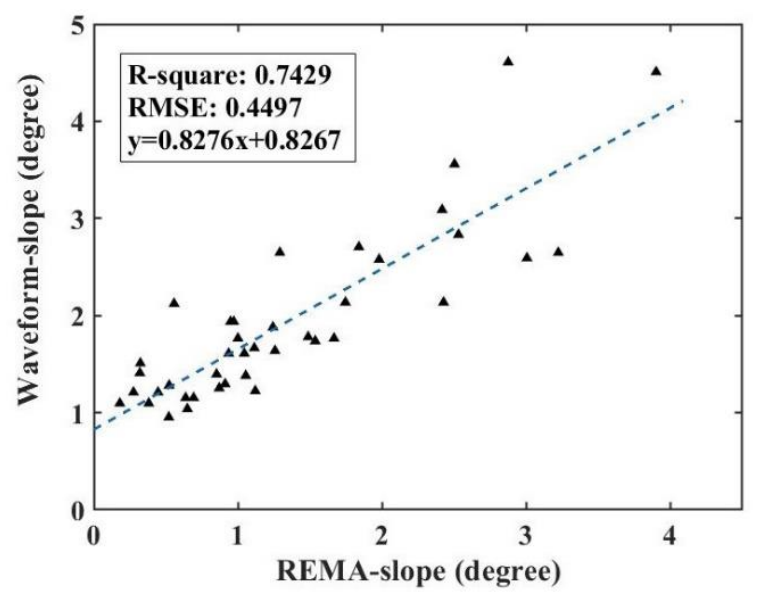

Figure 3. Scatter plot of the REMA slope versus the waveform slope and the corresponding regression model

To summarize, this paper extracts the surface slopes using ICESat/GLAS waveform data and REMA DEM data. A comparison is done between two sets of extracted slopes. The results show that there is a systematic bias between two extracted slope. The limitation of the method which extract slope from the waveform data is that it can only be used in moderately sloping surface. In the future, with the improvement of waveform data processing and simulating, as well as the improvement of the model for calculating surface slope, the results will be more accurate.

\section{ACKNOWLEDGEMENTS}

This work was supported by the National Natural Science Foundation of China under Grant No. 41822106 and 41571407, National High
Resolution Ground Observation System of China (GF-7) under Grant No. 11-Y20A12-9001-17/18, Science and Technology Innovation Action Plan Program of Shanghai under Grant No. 18511102100, Dawn Scholar of Shanghai under Grant No. 18SG22, and the Fundamental Research Funds for the Central Universities of China.

\section{REFERENCES}

[1] H. J. Zwally et al., "ICESat's laser measurements of polar ice, atmosphere, ocean, and land," Journal of Geodynamics, 34(3-4): 405-445 (2002)

[2] X. W. Wang et al., "Earth science applications of ICESat/GLAS," International Journal of Remote Sensing 32(23): 8837-8864 (2011)

[3] S. Nie et al., "A Novel Model for Terrain Slope Estimation Using ICESat/GLAS Waveform Data," Ieee Transactions on Geoscience and Remote Sensing, 56(1): 217-227 (2018)

[4] C. Mahoney et al., "Slope Estimation from ICESat/GLAS," Remote Sensing, 6(10): 10051-10069 (2014)

[5] D. H. Yi et al., "ICESat measurement of Greenland ice sheet surface slope and roughness," Annals of Glaciology, 42: 83-89 (2005)

[6] I. M. Howat et al., "The Reference Elevation Model of Antarctica," Cryosphere, 13(2): 665-674 (2019)

[7] V. H. Duong, "Processing and Application of ICESat Large Footprint Full Waveform Laser Range Data," (2010)

[8] A. C. Brenne et al., "Geoscience laser altimeter system algorithm theoretical basis document: Derivation of range and range distributions from laser pulse waveform analysis. Algorithm Theoretical Basis Documents (ATBD)," (2003)

[9] X. L. Li et al., "Terrain slope estimation within footprint from ICESat/GLAS waveform: model and method," Journal of Applied Remote Sensing, 6: 24 (2012) 